\title{
Tabvla Gratvlatoria
}

S. Eminêneia o Senhor D. António Ribeiro, Cardeal Patriarca de Lisboa e Magno Chanceler da UCP

S. Ex. ${ }^{a}$ Rev. ${ }^{\text {ma }}$ o Senhor Prof. Doutor D. José da Cruz Policarpo, Magnífico Reitor da UCP

S. Ex. ${ }^{\mathrm{a}}$ Rev. ${ }^{\mathrm{ma}}$ o Senhor D. António Monteiro, Bispo de Viseu

S. Ex. ${ }^{\mathrm{a}}$ Rev. ${ }^{\mathrm{ma}}$ o Senhor D. José Pedro da Silva, Bispo Emérito de Viseu

Prof. Doutor Padre José do Patrocínio Bacelar e Oliveira, Reitor Honórario da UCP

Prof. Doutor Valentim Xavier Pintado, Vice-Reitor da UCP

Prof. Doutor Padre Pio Alves de Sousa, Vice-Reitor da UCP

Dr. a Adília Moutinho Alarcão

Mestre Adriana Alves de Paula Martins

Prof. Doutor Aires Pereira do Couto

Cónego Dr. Abel Guimarães Figueiral

Prof. Doutor Alberto da Trindade Martinho

Prof. Doutor Alberto Coraceiro de Castro

Dr. Alberto Henrique de Figueiredo Lopes

Dr. Alberto Metelo Coimbra

Prof. Doutor Padre Albino de Almeida Matos

Dr. Alfredo Cabral

Prof. Doutor Padre Alfredo Dinis

Dr. Américo Nunes

Dr. Amílcar Araújo

Dr. ${ }^{a}$ Ana Cristina Rui Lopes de Almeida

Dr. ${ }^{a}$ Ana Maria Leite

Dr. ${ }^{a}$ Ana da Piedade Guerreiro M. Elias Pinheiro

Mestre Ana Forte

Dr. ${ }^{a}$ Ana Paula Barroco Lopes 
Dr. ${ }^{a}$ Ana Paula Pedroso Fernandes

Dr. ${ }^{\text {a }}$ Andrea da Silva Amaral

Dr. ${ }^{a}$ Ângela Alzira Brito

Dr. Ângelo Fernando Rodrigues

Doutor Anthony David Barker

Dr. António A. Huet Gonçalves

Dr. António Augusto Fernandes

Dr. António da Costa Moreira

Prof. Doutor António Luciano de Sousa Franco

Mestre António Manuel Antunes Rafael Amaro

Dr. António Joaquim Almeida Henriques

Dr. António Manuel Alves Henriques

Dr. António Manuel M. Cachulo Trindade

Prof. Doutor António Manuel P. Matoso Martinho

Prof. Doutor A. Morão Dias

Dr. António Pascoal de Sousa

Prof. Doutor António Pedro de Pinho Duarte Silva

Dr. António Soares Marques

Dr. Armando José Bernardo de Lacerda e Silva

Prof. Doutor Armando Luís Carvalho Homem

Prof. Doutor Augusto Guimarães de Medina

Dr. Baptista Rodrigues

Dr. Carlos Belchior

Dr. Carlos Duarte Coelho Peixeira Marques

Dr. Carlos Ferreira dos Santos

Mestre Carlos Jorge Videira Martins

Dr. Carlos Ricardo dos Santos Ferreira Moita

Mestre Célia dos Prazeres Ribeiro

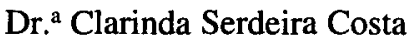

Dr. Custódio Lopes dos Santos

Dr. ${ }^{a}$ Cynthia Margaret Butterworth Fernandes

Dr. a Danielle Marie Berthe Georgette Foucaut

Prof. Doutor Domingos J. Cruz

S. Ex. ${ }^{a}$ Rev. ${ }^{\text {ma }}$ o Senhor D. Eurico Dias Nogueira, Arcebispo Primaz de Braga

Dr. ${ }^{a}$ Elisa Figueiredo

Mestre Fernando Baltazar Moreira Duarte

Mestre Fernando de Azevedo Andrade

Dr. Fernando Bettencourt Gaspar

Sr. Fernando Guedes

Dr. Fernando Joaquim Ferreira Mendes

10 Eng ${ }^{\circ}$. Fernando Jorge Mendonça Mota 
Dr. Fernando José Rodrigues F. Carvalho

Prof. Doutor Fernando Pacheco

Dr. Fernando Paulo do Carmo Baptista

Dr. Fernando Ruas

Dr. Filipe Almeida Santos

Prof. Doutor Francisco Carvalho Guerra

Prof. Doutor Francisco São José de Oliveira

Dr. Frank Leppert

Prof. Doutor Germano Marques da Silva

Dr. ${ }^{a}$ Helena Maria Ribeiro Almeida Costa

Prof. ${ }^{a}$ Doutora Helena Maria Simões Ferreira

Mestre Henrique Mota

Dr. Hermínio Ferreira

Dr. Hugo Coimbra

Dr. ${ }^{a}$ Ilda Nevado

Padre Dr. Ilídio Pinto Leandro

Dr. ${ }^{a}$ Isabel M. ${ }^{a}$ Carvalho Pinto Neves Aires Matos

Dr. ${ }^{a}$ Isabel Teresa Morais F. Campos Gil

S. Ex. ${ }^{\mathrm{a}}$ Rev. ${ }^{\text {ma }}$ o Senhor D. João Alves, Bispo de Coimbra

Prof. Doutor João Duarte Lourenço

Prof. Doutor João Fernandes Rebelo

Prof. Doutor João Luís César das Neves

Prof. Doutor João Luís Inês Vaz

Prof. João Pedro de Barros

Mestre João Manuel Vilas-Boas da Silva

Dr. Joaquim Cunha Braamcamp de Mancelos

S. Ex. ${ }^{a} \operatorname{Rev}^{\mathrm{ma}}$ o Senhor D. Joaquim Gonçalves, Bispo de Vila Real

Prof. Doutor Joaquim Marques Alves Fonseca

Dr. Joaquim Pereira Mateus

Mestre John Mckenny

Dr. Jorge Adolfo de Meneses Marques

Prof. Doutor Jorge Alves Osório

Prof. Doutor Jorge Costa André

Mestre Jorge Humberto dos Santos Seabra

Dr. José Sarmento Moniz

Prof. Doutor José Amado da Silva

Mestre José Augusto Pereira Sotto Mayor Pizarro

Doutor José Carlos Seabra Pereira

Cónego José Fernandes Vieira

Cónego Prof. Doutor Padre José Geraldes Freire

Prof. Doutor José M. Amado Mendes 
Prof. Doutor José Manuel Toscano Rico

Prof. Doutor José Manuel Vaz Caldas

Prof. Doutor José Ribeiro Ferreira

Dr. ${ }^{a}$ Josephine Gay Adamson Cameirão

S. Ex. ${ }^{\text {a Rev. }}{ }^{\text {ma }}$ D. Júlio Tavares Rebimbas, Bispo do Porto

Dr. Júlio Miguel Alves

Dr. ${ }^{\text {a Karen Bennet }}$

Dr. ${ }^{a}$ Leonor Cardoso

Dr. Leopoldo Sousa Camarinha

Dr. a Lúcia Laginhas

Prof. Doutor Lúcio Craveiro da Silva

Prof. Doutor Luís Araújo

Dr. Luís Filipe Coutinho Gomes

Dr. Luís Manuel Nogueira de Almeida

Dr. Luís Manuel Pereira Felisberto

Eng. Luís Manuel Pinto Ferreira Costa

Dr. Luís Pedro Figueiredo A. Santos Pinto

Prof. Doutor Manuel Braga da Cruz

Dr. Manuel Cerejeira Abreu Carneiro

S. Ex. ${ }^{\text {a }}$ Rev. ${ }^{\text {ma }}$ o Senhor D. Manuel d' Almeida Trindade, Bispo Emérito de Aveiro

Dr. Manuel Chaves de Andrade

Prof. Doutor Manuel Lopes Porto

Padre Dr. Manuel da Rocha Felício

Prof. Doutor Manuel de Oliveira Pulquério

Mestre Henrique Maximino de Almeida

Dr. Manuel Hernâni Barros Gomes Vallera

Prof. Doutor Manuel Farinha dos Santos

Prof. Doutor Padre Manuel Isidro Araújo Alves

Prof. Doutor Manuel Maria de Melo Alte da Veiga

Dr. Manuel Santiago e Costa

Prof. a Doutora Maria Aparecida Ribeiro Pinho

Dr. a Maria Cristina Nascimento R. M. A. Sousa Gomes

Dr. a Maria da Graça Ferreira

Dr. ${ }^{a}$ Maria de Fátima dos Prazeres Eusébio

Prof. a Doutora Maria de Fátima Sousa e Silva

Dr. ${ }^{a}$ Maria de Jesus Quintas Reis Cabral

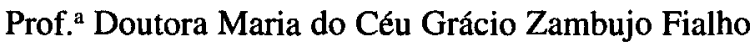

Mestre Maria do Céu Lopes

Dr. ${ }^{a}$ Maria Eugénia Duarte Figueiredo Fernandes

Dr. a Maria Filomena Dias Capucho

12 Prof. ${ }^{\text {a }}$ Doutora Maria Hermínia Deulonder C. Amado Laurel 
Dr. ${ }^{a}$ Maria Luísa Alves Ferreira de Almeida

Prof.a Doutora Maria Olívia Dias

Dr. ${ }^{a}$ Maria Teresa Ferreira Gomes Loureiro Filipe

Prof. Doutor Mário Júlio de Almeida Costa

Prof. Doutor Dário Varela Gomes

Prof. Doutor Martim Manuel Portugal Ferreira

Prof. Doutor Nicolau de Almeida Vasconcelos Raposo

Dr. ${ }^{a}$ Paula Cristina Fragão Pereira

Prof. Doutor Paulino de Freitas Teixeira

Dr. Paulo Alexandre Oliveira Castro Ribeiro

Dr. Paulo Renato Martins Lourenço

Dr. Pedro Paraíso

Dr. Pedro Soveral de Carvalho

Cónego Dr. Orlando Soares de Paiva

Eng. Roberto Carneiro

Mestre Rogério Morais Teixeira

Mestre Rogério Paulo Garcia S. P. Matias

Dr. ${ }^{a}$ Rosa Maria Soares Campos M. Couto

Dr. Rubens Mourão Terra

Prof. Doutor Rui Alberto Ferreira Santos Alves

Dr. Rui Luís Vide da Cunha Martins

Dr. ${ }^{a}$ Sara Manuela Ribeiro Martins Augusto

Prof. Doutor Sebastião José Formosinho Sanches

Prof. Doutor Sebastião Tavares de Pinho

S. Ex. ${ }^{a}$ Rev ${ }^{\text {ma }}$ D. Serafim de Sousa Ferreira e Silva, Bispo de Leiria

Prof. Doutor Telmo dos Santos Verdelho

Prof. Doutor Victor Augusto Mendes dos Santos

Prof. Doutor Victor Oliveira Jorge

Dr. Sérgio Tormenta

Dr. ${ }^{a}$ Sofia Leitão Borges

Prof. ${ }^{a}$ Doutora Susana Oliveira Jorge 